\title{
Does Logarithm Transformation of Microarray Data Affect Ranking Order of Differentially Expressed Genes?
}

\author{
Wentian Li, Young Ju Suh, Jingshan Zhang
}

\begin{abstract}
A common practice in microarray analysis is to transform the microarray raw data (light intensity) by a logarithmic transformation, and the justification for this transformation is to make the distribution more symmetric and Gaussian-like. Since this transformation is not universally practiced in all microarray analysis, we examined whether the discrepancy of this treatment of raw data affect the "high level" analysis result. In particular, whether the differentially expressed genes as obtained by $t$-test, regularized $t$-test, or logistic regression have altered rank orders due to presence or absence of the transformation. We show that as much as $20 \%-40 \%$ of significant genes are "discordant" (significant only in one form of the data and not in both), depending on the test being used and the threshold value for claiming significance. The $t$-test is more likely to be affected by logarithmic transformation than logistic regression, and regularized $t$-test more affected than $t$-test. On the other hand, the very top ranking genes (e.g. up to top 20-50 genes, depending on the test) are not affected by the logarithmic transformation.
\end{abstract}

\section{INTRODUCTION}

The number of copies of single-stranded messenger-RNA (mRNA) can be used to infer the amount of protein product produced by certain gene, and is called the "expression level". Ideally, one would like to count the number of copies of certain mRNA directly. But in microarray chips, the amount of a specific mRNA is measured indirectly by the emission of fluorescence light. It is necessary to transform the raw data of light intensity obtained by optical detection to a summarized quantity that indicates the expression level. Deriving the expression level from raw data is called the "low-level" analysis, and it can be complicated by the details of the technology and chip platform [1], [2]. Reaching conclusions such as the determination of differentially expressed genes using the expression level data is called the "highlevel" analysis.

After the expression level is derived from the raw data, another preprocessing step is commonly practiced: log-transformation. The standard motivation for the logtransformation is that the distribution of the derived expression level is typically asymmetric with long tail at the high expression end. Many parametric statistical tests require variables to follow a Gaussian/normal distribution.

W. Li is a Research Scientist with the Robert $\mathrm{S}$ Boas Center for Genomics and Human Genetics, Feinstein Institute for Medical Research, North Shore LIJ Health System, Manhasset, NY 11030, USA wli@nslij-genetics.org

Y.J. Suh is a Research Professor of The Research Institute of Natural Sciences, Sookmyung Women's University, Seoul 140-742, Korea. yjsprite@yahoo.co.kr

J. Zhang is a Senior Statistician at Forest Research Institute, Jersey City, NJ 07311, USA jingshan.zhang@frx.com
The log-transformation is an attempt to convert an asymmetric distribution to a symmetric and Gaussian-like one. Other transformations for the purpose of "normality" are also possible [3], such as square-root, Box-Cox [4], and arcsine transformations. In microarray data, transformations were proposed along the line of variance stabilization [5], [6]

A novel alternative explanation of the use of logtransformation might be that human perceive brightness of light as the logarithm of light energy, similar to our perceiving loudness of sound as the logarithm of sound intensity. In general, all human perception of physical stimuli is proportional to the logarithm of amount of stimuli, under the names of Weber-Fechner's law [7], [8] and Steven's law [9]. For the light-intensity-derived expression level, logtransformation can be viewed as a way to measure the "perception signal" from the data.

From the statistical point of view, logarithm transformation can take down an outlier with extreme high value, thus affecting the group mean. On the other hand, logarithm transformation or any 1-to-1 transformation will not shuffle the relative order of expression values, thus will not affect a rank-based test result such as Wilcoxon-Mann-Whitney test [10]. For a specific test or statistical model, the effect of log-transformation on the result is not clear, even though we know it has no effect if the test is rank-based, and has some effects if there are outliers. For linear classifiers, the violation of Gaussian distribution affect some methods more (e.g. Fisher's linear discriminant analysis, perceptron) but less so on other methods (e.g., logistic regression, support vector machine) [11].

Another note on investigating the effect of logtransformation is that one can focus either on the whole list of genes, or only on the more interesting top ranking genes. For example, with a log-transformation, the top 1 and 2 differentially expressed genes may be switched while the rank of all other genes are unchanged. Even though the effect of log-transformation on the whole list of genes could be small, the minor rearrangement of the top ranking genes can be crucial in designing the subsequent experiments such as gene validation by real-time PCR.

We will examine the effect of log transformation on two or three simple methods for selecting differentially expressed genes on a real microarray dataset. Log-transformation is just one factor that change the apparent value of data, there are other factors as well such as the normalization procedure during the "low-level" analysis, change of the probe set design, change of the microarray platform, etc. 


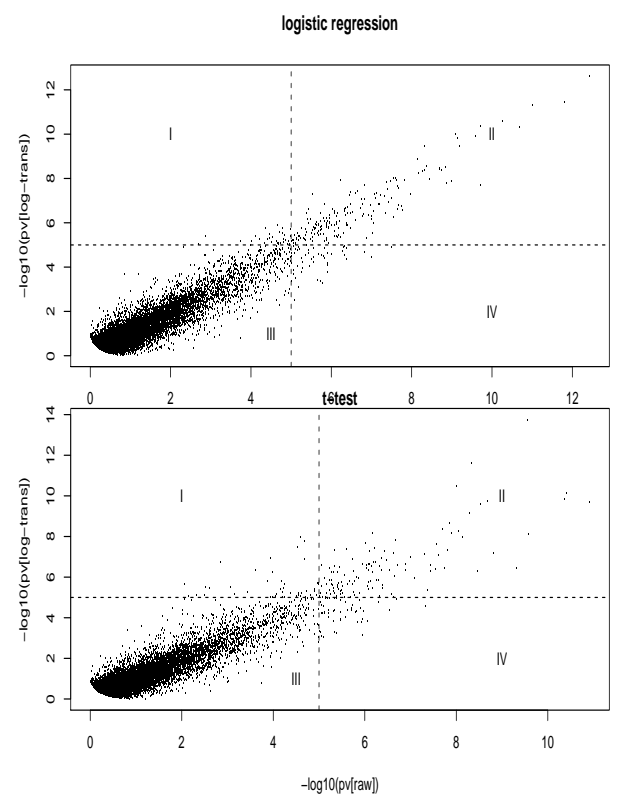

Fig. 1. Minus $\log$ of $p$-values of tests on $\log$ transformed vs. original data. The $x$ axis is $-\log _{10}$ ( $p$-value) for the original expression data, and $y$ axis is $-\log _{10}$ ( $p$-value) for the log-transformed data. The top plot is for logistic regression and bottom plot for $t$-test. The four quadrants as split by $x=5$ and $y=5$ are indicated. Each point represents a gene.

\section{METHODS AND DATA}

\section{A. Student's t-test}

The Student's $t$-test is used here as a representative of tests that make assumption on variable normality. We expect the normality requirement is met better for the log-transformed data than the original data. The $t$-statistic is defined as the ratio of the difference of two group means and the standard error of this difference: $t=\left(E_{1}-E_{2}\right) / \sqrt{s_{1}^{2} / n_{1}+s_{2}^{2} / n_{2}}$, where $E_{1,2}, s_{1,2}^{2}, n_{1,2}$ are the mean, variance, and sample size of group 1 and 2 . The $p$-value given a $t$-statistic value is determined by the Student's $t$-distribution with degree of freedom $d f$. Usually, $d f$ is equal to $n_{1}+n_{2}-2$, but when the variances in two groups are not equal, a more complicated formula for $d f$ can be used [12]. We use such a method as implemented in the $R$ statistical package (http://www.r-project.org).

\section{B. Logistic regression}

Logistic regression is used to represent statistical models which do not have a strong normality requirement. The advantage for models or tests lacking such a requirement is that these are more robust. The disadvantage for models without the normality requirement is that when the variable is in fact distributed as Gaussian, these are less "efficient" as classifiers [13]. The significance of a single-gene logistic regression can be determined by a likelihood-ratio test: (2) log-maximum-likelihood of the logistic regression model subtract that of a null model follows a $\chi^{2}$ distribution with one degree of freedom, under the null hypothesis. Thus given the (-2) log-likelihood ratio (called "deviance"), the $p$-value can be determined using the $\chi^{2}$ distribution.
TABLE I

PERCENTAGE OF DISCORDANT GENES: (I+IV)/(I+II+IV)

\begin{tabular}{|c|c|c|c|c|c|c|}
\multicolumn{1}{|c}{ logistic regression } \\
\hline$p_{0}$ & I+IV & II & $\%(95 \% \mathrm{CI})$ & I+IV & II & $\%(95 \%$ CI) \\
\hline $10^{-9}$ & 0 & 10 & $0 \%(0-0)$ & 7 & 4 & $64 \%(35-92)$ \\
$10^{-8}$ & 6 & 20 & $23(7-39)$ & 8 & 11 & $42(20-64)$ \\
$10^{-7}$ & 22 & 40 & $35(24-47)$ & 21 & 21 & $50(35-65)$ \\
$10^{-6}$ & 44 & 84 & $34(26-43)$ & 40 & 52 & $43(33-54)$ \\
$10^{-5}$ & 82 & 176 & $32(26-37)$ & 92 & 119 & $44(37-50)$ \\
$10^{-4}$ & 163 & 346 & $32(28-36)$ & 170 & 266 & $39(34-44)$ \\
0.001 & 328 & 709 & $32(29-34)$ & 345 & 593 & $37(34-40)$ \\
0.01 & 744 & 1698 & $30(29-32)$ & 771 & 1520 & $34(32-36)$ \\
\hline
\end{tabular}

\section{Regularized t-test and significance analysis of microar- rays (SAM)}

Since low expression level also leads to low variance, $t$ statistic can be high due to low expression level. Penalized or regularized statistics add an extra term $s_{0}$ to prevent this small variance from inflating the statistic: $d=\left(E_{1}-\right.$ $\left.E_{2}\right) /\left(\sqrt{s_{1}^{2} / n_{1}+s_{2}^{2} / n_{2}}+s_{0}\right)$. SAM (significance analysis of microarray) is a method for determining the value of $s_{0}$ [14]. SAM test statistic, $d$-score, was calculated by the SAM package obtained from http://Www-stat.stanford.edu/ tibs/SAM/

\section{Microarray data}

The illustrative microarray data is a profiling study of rheumatoid arthritis. There are 43 patients and 48 normal controls, which is more than the 29 patients and 21 controls used in the previous publication [15]. The mRNA was extracted from the peripheral blood mononuclear cells. The microarray data is obtained from the Affymetrix HG-U133A GeneChip with 22,283 genes/probe-sets, and was normalized by the Affymetrix microarray suite (MAS) program.

\section{RESULTS}

\section{A. Proportion of discordant differentially expressed genes}

Fig 1 shows the minus log of $p$-values of log-transformed expression data vs that of un-log-transformed (raw) expression data, for both logistic regression (top) and $t$-test (bottom). Taking all genes as a whole, the two sets of $p$-values are highly correlated (correlation coefficients are 0.94 and 0.93 , respectively). In order to highlight the differences, especially for the high-ranking differentially expressed genes, we split the plot into four quadrants by a vertical line at $x=a$ and horizontal line at $y=a$. The parameter $a=-\log _{10}\left(p_{0}\right)$ corresponds to gene selection threshold $p_{0}$ for $p$-values. For example, the $a=5$ in Fig 1 corresponds a $p$-value threshold of $p_{0}=0.00001$.

The genes in quadrants I, II, and IV have at least one $p$ value of the two (log and raw data) smaller than $p_{0}$, whereas the genes in quadrant II have both $p$-values smaller than $p_{0}$. If log-transformation has no effect on the gene selection, there will be no points in quadrants I and IV. We use the percentage of points in I and IV out of all points in I,II, IV as a measure of the inconsistency between the test results on raw and log-transformed data. If points in quadrants I and IV are 

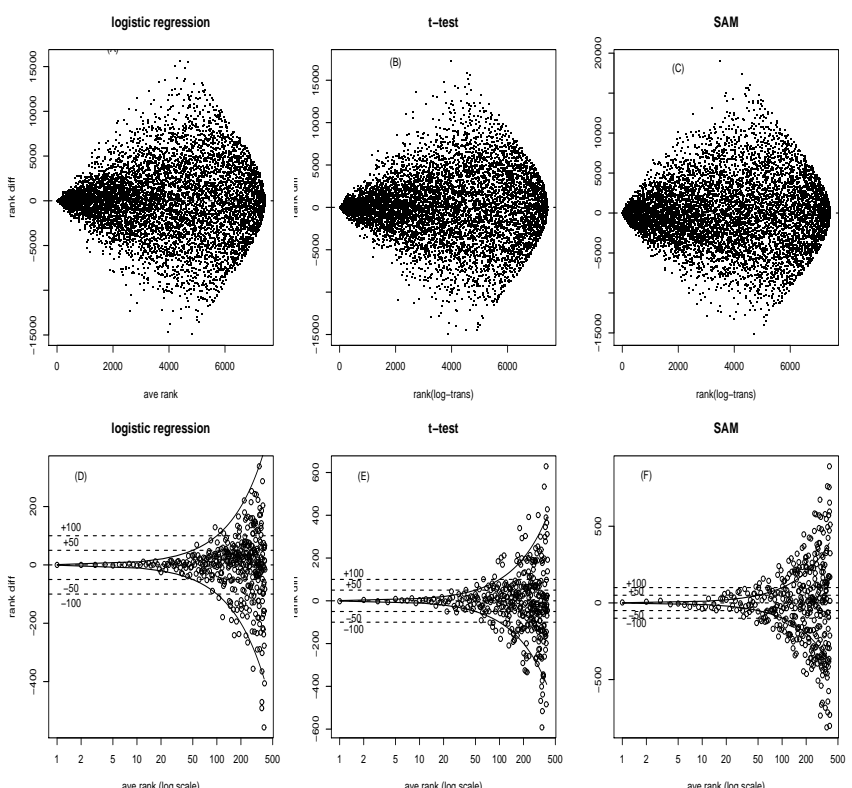

Fig. 2. Rank difference $d$ as a function of averaged rank $R_{a}$ for all 22283 genes $(\mathrm{A}, \mathrm{B}, \mathrm{C})$ and for top-400 genes (D,E,F). Both rank difference $d$ and averaged rank $R_{a}$ concern the same gene on two different types of data (raw and log-transformed). (A) and (D) are results for logistic regression, (B) and (E) are for $t$-test, (C) and (F) for SAM. The $x$-axis in $(\mathrm{D}, \mathrm{E}, \mathrm{F})$ is in log scale to highlight the top-ranking genes. In (D,E,F), $d=50,-50,100,-100$ and $d=R_{a}, d=-R_{a}$ lines are drawn.

called "discordant" and those in quadrant II "concordant", this measure is the percentage of discordant genes among all differentially expressed genes by either one type of data.

Table 【 shows the discordant percentage and their $95 \%$ confidence intervals $(\mathrm{CI})$ at various gene selection threshold $p_{0}\left(=10^{-9}, \cdots, 10^{-4}, 0.001,0.01\right)$. As expected, the $t$-test result is more affected by the log transformation than logistic regression: at all $p_{0}$ threshold values, the percentage of discordant differentially expressed genes is higher in $t$-test than in logistic regression. The average discordant percentage at eight $p_{0}$ values is $27 \%$ for logistic regression and $44 \%$ for $t$-test.

It was however surprising that for logistic regression, except for the extremely differentially expressed genes (e.g., when $p$-value $<10^{-9}$, the discordant percentage is zero), the discordant percentage is not negligible. If either one of the raw or log-transformed data is used for logistic regression analysis, as much as $10 \%-20 \%$ of the claimed differentially expressed genes will not be claimed so by another data.

\section{B. Ranking change due to log transformation}

The effect of log-transformation can also be examined by the ranking of a gene in both datasets. If log-transformation has no effect, the rank of a gene by (e.g.) $p$-value will be unchanged. We use the notation $R_{n}(i), R_{l}(i)$ for the rank of gene- $i$ in the raw and log-transformed data, and define $R_{a}(i)$ as the average of the two: $R_{a}(i) \equiv\left(R_{n}(i)+R_{l}(i)\right) / 2$, and $d(i)$ as the rank difference: $d(i)=R_{n}(i)-R_{l}(i)$. Fig 2 (A,B,C) show $d$ vs. $R_{a}$ for logistic regression, $t$-test, and SAM (genes are ranked by absolute value of the $d$-score) for all 22283 genes.
Fig $2(\mathrm{~A}, \mathrm{~B}, \mathrm{C})$ indicate that for the whole gene set there is a similar pattern for all three test-statistics: for high- and lowranking genes, they are high and low ranked in both raw and log-transformed data (thus smaller rank differences). As the majority of genes are not differentially expressed, the overall scattering pattern in Fig 2 $(\mathrm{A}, \mathrm{B}, \mathrm{C})$ may not be as interesting as the behavior near the high-ranking differentially expressed genes.

To focus on the top-ranking genes, Fig 2 (D,E,F) zoom in for the top-400 genes ( $x$-axis is in log scale). First, we notice that for the very top genes (e.g. up to top-10), the ranking is unchanged or changed very little by the log transformation in any one of the three tests/models. Second, $t$-test has reached rank-difference of $d=50$ and $d=100$ sooner (i.e., at a higher ranking) than logistic regression, reconfirming our previous conclusion that $t$-test is more likely to be affected by $\log$ transformation than logistic regressions. Using the $d=R_{a}$ and $d=-R_{a}$ envelope, we see that points are more likely to be outside the envelopes for $t$-test than the logistic regression. The third observation is that SAM test result is affected even more by log transformation than $t$-test. In Fig 2 (F), many points are far outside the envelope region.

\section{CONCLUSIONS AND FUTURE WORKS}

\section{A. Conclusions}

Using one microarray dataset, we have shown that $\log$ transformation may affect results on selecting differentially expressed genes. If we call all genes that are significant by tests on either raw or log-transformed data "differentially expressed genes", and those genes that are significant in test of only one of the two types of data "discordant", the discordant as a proportion of the all (discordant and concordant) differentially expressed genes can be as high as $27 \%$ for logistic regression and $44 \%$ for $t$-test. The larger discordant percentage for $t$-test confirms our general understanding that tests that require variable normality are more likely to be affected by variable transformation.

\section{B. Future Works}

We plan to extend the results here to other public domain microarray datasets and to other tests, models, and measures for determining differentially expressed genes.

\section{ACKNOWLEDGMENTS}

We thank Franak Batliwalla for providing the data.

\section{REFERENCES}

[1] C. Li, W.H. Wong, "Model-based analysis of oligonucleotide arrays: Expression index computation and outlier detection", Proc. Nat. Acad. Sci., vol 98, pp.31-36.

[2] R.A. Irizarry, B.M. Bolstad, F. Collin, L.M. Cope, B. Hobbs, T. P. Speed, "Summaries of Affymetrix GeneChip probe level data", Nucl. Acids Res., vol 31, 2003, e15.

[3] R.R. Sokal, F.J. Rohlf, Biometry, 3rd edition, W.H. Freeman and Co., New York; 1995.

[4] G.E.P. Box, D.R. Cox , "An analysis of transformations", J. R. Stat Soc. B, vol 26, 1964, pp.211-243.

[5] B.P. Durbin, J.S. Hardin, D.M. Hawkins, D.M. Rocke, "A variancestabilizing transformation for gene-expression microarray data", Bioinformatics, vol 18(suppl 1), 2002, pp.S105-S110. 
[6] B. Durbin, D.M. Rocke, "Estimation of transformation parameters for microarray data", Bioinformatics, vol 19, 2003, pp.1360-1367.

[7] E.H. Weber, De pulsi, resorptione, auditu ert tactu. Annotationes anatomicae et physiologicae, C.F. Löhler, Leipzig; 1834.

[8] G.T. Fechner, Elemente der Psychophsik, Breitkopf \& Härtel, Leipzig; 1860.

[9] S.S. Stevens, "On the psychophysical law", Psychol. Rev., vol 64, 1957, pp.153-181.

[10] H.B. Mann, D.R. Whitney, "On a test of whether one of 2 random variables is stochastically larger than the other", Ann. Math. Stat. , vol 18, 1947, pp.50-60.

[11] T. Hastie, R. Tibshirani, J. Friedman, The Elements of Statistical Learning, Springer, New York; 2001.

[12] B. L. Welsh, "The generalization of 'Student's' problem when several different population variances are involved", Biometrika, vol 34, 1947, pp.28-35.

[13] B. Efron, "The efficiency of logistic regression compared to normal discriminant analysis", J. Am. Stat. Asso., vol 70, 1975, pp.892-898.

[14] V. Tusher, R. Tibshirani, C. Chu, (2001): "Significance analysis of microarrays applied to the ionizing radiation response", Proc. Natl. Acad. Sci., vol 98, 2001, pp. 5116-5121.

[15] F.M. Batliwalla, E.C. Baechler, X. Xiao, W. Li, S. Balasubramaniuan, H. Khalili, A. Damle, W.A. Ortmann, A. Perrone, A.B. Kantor, P.S. Gulko, M. Kern, R. Furie, T. W. Behrens, P. K. Gregersen, "Peripheral blood gene expression profiling in rheumatoid arthritis", Gene and Immunity, vol 6, 2005, pp. 388-397. 